\title{
Call out to community dentists
}

The BDA is calling on community dental services (CDS) dentists to give their experience of working in services across the UK to help inform our yearly submission to the Government's review body on dentists' pay.

The questionnaire aims to investigate the morale, motivation and levels of job satisfaction among community dentists and seeks to learn more about their working conditions and views on the services they work in. The results will also help to build a picture of the impact of ongoing changes on the workforce and community dental service.

All dentists working in the CDS are welcome to participate. If you have been emailed an invitation to take part, or received a questionnaire in the post, please complete the questionnaire by Friday 13 July and help us to be the collective voice of dentistry. Non-BDA members who are interested in giving their views can do so at http://www. smartsurvey.co.uk/s/BDA_CDS_2018/.

To thank members for their time, those who respond using their personalised questionnaire will be entered into a prize draw to win a $£ 100$ voucher. Please be assured that your information will be anonymous and we will not pass your details on to any third parties.

\section{BOOK REVIEW}

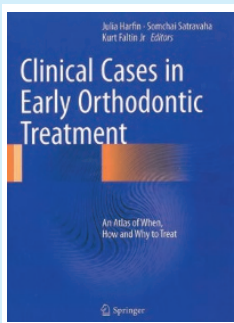

\section{CLINICAL CASES IN EARLY ORTHODONTIC TREATMENT: AN ATLAS OF WHEN, HOW AND WHY TO TREAT}

Julia Harfin, Somchai Satravaha and Kurt Faltin Jr

Springer, 2017

price $f 199.99$ pp. 351

ISBN: 9783319462509

This book is written by three authors who are currently based in Argentina, Thailand and Brazil. It provides a series of clinical cases describing common malocclusions in children. It describes the management of these cases, with an emphasis on early intervention in the form of interceptive treatment. Within these cases they address the issue of one-phase or two-phase treatment, considering the severity and aetiology. Within each chapter the authors discuss different alternatives for each malocclusion in a step-by-step manner. They discuss the importance of the timing of treatment, growth and normalisation of the functional problems.

The cases are laid out in chapters, although there are no sub-headings within each chapter which can make navigation a little tricky. The chapters discuss the following topics: the treatment of atypically rotated central incisors; early management of periodontal problems; correction and maintenance of the position of the first upper molar; early treatment of class II and class III malocclusions; use of lingual brackets; management of dental asymmetries; TMJ problems in children; early treatment of open bite problems; and the Bennun-Harfin protocol for interdisciplinary oral cleft treatment. Each chapter is enhanced by a comprehensive bibliography, numerous clinical photographs, radiographs and the long-term results of the cases. Although some of the photograph and radiograph quality is poor with images being blurry and not always in focus. This may be due to the images being enlarged for publication.

Although it is a useful, informative text looking at some interesting cases, the image quality does not complement the text and is distracting for the reader. As it is the first edition of the book, I would recommend that the second edition contains improved quality images to facilitate the reader. It is not an evidence-based text and is purely based on clinical cases of the authors. Therefore, I would presume that it will not be high on the reading lists of orthodontic trainees or orthodontists, but does provide a wealth of clinical information. 